At present very few data are available on $M 1$ transitions in $4 N+2$ nuclei, so that an upper limit on the supermultiplet impurity cannot be determined in the same way as for ${ }^{6} \mathrm{Li}$. However, there is good reason to believe that the impurities for ${ }^{10} \mathrm{~B}^{*}$ and ${ }^{18} \mathrm{~F}$ are small since these two nuclei can be described as two nucleons in a triplet state coupled to ${ }^{8} \mathrm{Be}$ and ${ }^{16} \mathrm{O}$ cores, respectively, and it is known ${ }^{1}$ that the ground states of ${ }^{8} \mathrm{Be}$ and ${ }^{16} \mathrm{O}$ are nearly pure scalar supermultiplets. Thus, inequalities (6), (7), and (12) are still valid and one gets

$$
\begin{aligned}
& 0.65 \mu_{N} \leqslant \mu\left({ }^{10} \mathrm{~B}^{*}\right) \leqslant 0.88 \mu_{N}, \\
& 0.66 \mu_{N} \leqslant \mu\left({ }^{18} \mathrm{~F}\right) \leqslant 0.88 \mu_{N} .
\end{aligned}
$$

We note that a recent theoretical calculation ${ }^{6}$ gives $\mu\left({ }^{18} \mathrm{~F}\right)=1.14 \mu_{N}$, in disagreement with the above upper limit.

In concluding, I would like to point out that more accurate experimental data on $\Delta T=1 \mathrm{GT}$ and $M 1$ transitions from the ground to all excited states in doubly odd $4 N+2$ nuclei will give a good estimate of the supermultiplet impurities of the ground state, thus enabling us to establish the validity of SU(4) in these nuclei.

The author would like to thank Professor L. A. Radicati for his interest in this work and Professor D. Fotiadi and Professor T. N. Truong for their hospitality extended to him at the Centre.

Financial support from the Centre de Physique Théorique de l'Ecole Polytechnique is gratefully acknowledged.

*Equipe de Recherche associée au Centre National de la Recherche Scientifique.

${ }^{1}$ Pham T. N., Nucl. Phys. A185, 413 (1972).

${ }^{2}$ For a discussion of supermultiplet theory, see, for example, L. A. Radicati, in Application of Mathematics to Problems in Theoretical Physics, edited by F. Lurçat (Gordon and Breach, New York, 1967), and other references cited therein.

${ }^{3}$ R. W. Kavanagh, Nucl. Phys. A129, 172 (1969).

${ }^{4}$ J. N. Bahcall, Nucl. Phys. $75, \overline{10}(1966)$.

${ }^{5}$ R. Avida, I. Ben-Zvi, G. Goldring, S. S. Hanna, P. N. Tandon, and Y. Wolfson, Nucl. Phys. A182, 359 (1972).

${ }^{6}$ M. R. Gunye and S. B. Khadkikar, Phys. Rev. C $\underline{4}, 1073$ (1971).

\title{
Rotating Black Holes: Separable Wave Equations for Gravitational and Electromagnetic Perturbations*
}

\author{
Saul A. Teukolsky \\ California Institute of Technology, Pasadena, California 91109
}

(Received 10 July 1972)

\begin{abstract}
Separable wave equations with source terms are presented for electromagnetic and gravitational perturbations of an uncharged, rotating black hole. These equations describe the radiative field completely, and also part of the nonradiative field. Nontrivial, source-free, stationary perturbations are shown not to exist. The barrier integral governing synchrotron radiation from particles in circular orbits is shown to be the same as for scalar radiation. Future applications (stability of rotating black holes, "spin-down," superradiant scattering, floating orbits) are outlined.
\end{abstract}

It is generally accepted that the gravitational collapse of a massive rotating star can produce a rotating black hole. Moreover, black holes may play important roles in a number of astrophysical phenomena: (i) One or more black holes near the center of the Galaxy might be the origin of Weber's ${ }^{1}$ gravitational-wave events; (ii) a massive black hole at the center of the Galaxy has been postulated ${ }^{2}$ to explain radio and infrared phenomena there; (iii) the $\mathrm{x}$-ray source Cyg-XI -and also $2 \mathrm{U}-0900-40$-is likely to be a black hole in close orbit around a $B$-type supergiant star, with the $x$ rays emitted by gas flowing from star to hole. ${ }^{3}$

These developments create an urgent need for two types of black-hole calculations: first, cal- 
culations on matters of principle, such as whether rotating black holes are unstable against spontaneous loss of rotational energy and angular momentum; second, realistic astrophysical calculations of black-hole processes. Both types of calculations are amenable to perturbation techniques, where the dynamics of matter, electromagnetism, and gravitational waves takes place in the fixed background geometry of the black hole. Until now, calculations of electromagnetic and gravitational perturbations were restricted to the nonrotating (Schwarzschild) case, where the static, spherically symmetric background geometry guarantees a complete separation of variables in the perturbation equations. In the rotating case, the background geometry is described by the $\operatorname{Kerr}^{4}$ metric, and the electromagnetic ${ }^{5}$ and gravitational $l^{6}$ perturbation equations have appeared to be inseparable. However, the scalar wave equation $\square \Phi=4 \pi T$ is separable ${ }^{7,8}$ in Boyer-Lindquist ${ }^{9}$ coordinates, and has been used to make qualitative predictions about the electromagnetic and gravitational cases.

In this Letter, we present separable equations for the radiative parts of electromagnetic and gravitational perturbations. The equations are consolidated into a single master equation, Eq. (5) below, with a "spin-weight parameter" $s$ specifying the type of field under study (scalar, electromagnetic, gravitational) and the particular radiative part of the field involved. The derivations, which are similar to earlier approaches, ${ }^{10,11,5,6}$ use the Newman-Penrose ${ }^{12}$ formalism and will be published elsewhere. Sufficient information is given below to enable calculations to be performed.

In Boyer-Lindquist coordinates with $c=G=1$, the Kerr metric is

$$
d s^{2}=(1-2 M r / \Sigma) d t^{2}+\left(4 M a r \sin ^{2}(\theta) / \Sigma\right) d t d \varphi-(\Sigma / \Delta) d r^{2}-\Sigma d \theta^{2}-\sin ^{2}(\theta)\left(r^{2}+a^{2}+2 M a^{2} r \sin ^{2}(\theta) / \Sigma\right) d \varphi^{2} .
$$

Here $M$ is the mass of the black hole, $a M$ its angular momentum, $\Sigma=r^{2}+a^{2} \cos ^{2} \theta$, and $\Delta=r^{2}-2 M r+a^{2}$. We introduce Kinnersley's ${ }^{13}$ null tetrad, which has $[t, r, \theta, \varphi]$ components

$$
l^{\mu}=\left[\left(r^{2}+a^{2}\right) / \Delta, 1,0, a / \Delta\right], \quad n^{\mu}=\left[r^{2}+a^{2},-\Delta, 0, a\right] / 2 \Sigma, \quad m^{\mu}=[i a \sin \theta, 0,1, i / \sin \theta] / 2^{1 / 2}(r+i a \cos \theta) .
$$

The electromagnetic field is characterized by the Newman-Penrose components

$$
\varphi_{0}=F_{\mu \nu} l^{\mu} m^{\nu}, \quad \varphi_{1}=\frac{1}{2} F_{\mu \nu}\left(l^{\mu} n^{\nu}+m^{* \mu} m^{\nu}\right), \quad \varphi_{2}=F_{\mu \nu} m^{* \mu} n^{\nu},
$$

where $F_{\mu \nu}$ is the electromagnetic field tensor and an asterisk denotes complex conjugation. The components $\varphi_{0}$ and $\varphi_{2}$ are the "ingoing and outgoing radiative parts" of the field. Maxwell's equations in the fixed Kerr background with four-current $J^{\mu}$ lead directly to Eq. (5) below for $\varphi_{0}$ and $\varphi_{2}$.

Gravitational radiation is described by perturbations in the Weyl tensor $C_{\alpha \beta \gamma \delta}$ (which is equal to the Riemann tensor in vacuum). The "ingoing and outgoing radiative parts" of the Weyl tensor are

$$
\psi_{0}=-C_{\alpha \beta \gamma \delta} l^{\alpha} m^{\beta} l^{\gamma} m^{\delta}, \quad \psi_{4}=-C_{\alpha \beta \gamma \delta} n^{\alpha} m^{* \beta} n^{\gamma} m^{* \delta} .
$$

These quantities are invariant under gauge transformations and infinitesimal tetrad rotations. Firstorder perturbations of the Newman-Penrose version of the Einstein field equations, with material stress-energy tensor $T_{\mu \nu}$, lead to Eq. (5) below for $\psi_{0}$ and $\psi_{4}$.

The master perturbation equation is

$$
\begin{array}{r}
{\left[\frac{\left(r^{2}+a^{2}\right)^{2}}{\Delta}-a^{2} \sin ^{2} \theta\right] \frac{\partial^{2} \psi}{\partial t^{2}}+\frac{4 M a r}{\Delta} \frac{\partial^{2} \psi}{\partial t \partial \varphi}+\left[\frac{a^{2}}{\Delta}-\frac{1}{\sin ^{2} \theta}\right] \frac{\partial^{2} \psi}{\partial \varphi^{2}}-\Delta^{-s} \frac{\partial}{\partial r}\left(\Delta^{s+1} \frac{\partial \psi}{\partial r}\right)-\frac{1}{\sin \theta} \frac{\partial}{\partial \theta}\left(\sin \theta \frac{\partial \psi}{\partial \theta}\right)} \\
-2 s\left[\frac{a(r-M)}{\Delta}+\frac{i \cos \theta}{\sin ^{2} \theta}\right] \frac{\partial \psi}{\partial \varphi}-2 s\left[\frac{M\left(r^{2}-a^{2}\right)}{\Delta}-r-i a \cos \theta\right] \frac{\partial \psi}{\partial t}+\left(s^{2} \cot ^{2} \theta-s\right) \psi=4 \pi \Sigma T
\end{array}
$$

Table I specifies the field quantities $\psi$ which satisfy this equation, the corresponding values of the spinweight parameter $s$, and the source terms $T$. The source terms are written in Newman-Penrose notation.

Equation (5) can be separated by writing

$$
\psi=e^{-i \omega t} e^{i m \varphi} S(\theta) R(r)
$$

[Note that Eq. (5) is also separable in $\operatorname{Kerr}^{14}$ coordinates or any other coordinate system related to 
TABLE I. Field quantities $\psi$, spin-weight $s$, and source terms $T$ for Eq. (5).

\begin{tabular}{|c|c|c|c|c|}
\hline$\Psi$ & $\mathbf{s}$ & \multicolumn{3}{|c|}{ Source Term $\mathrm{T}$} \\
\hline$\Phi$ & 0 & $\square \Phi=4 \pi \mathrm{T}$ & ( $T$ is the scalar charge density) & \\
\hline $\begin{array}{l}\varphi_{0} \\
\rho^{-2} \varphi_{2}\end{array}$ & -1 & $\begin{array}{l}(\delta-2 \tau) \mathrm{J}_{\ell} \\
(\Delta+\mu) \rho^{-2}\end{array}$ & $\begin{array}{l}-\left(D-2 \rho-\rho^{*}\right) J_{m} \\
J_{m^{*}}-\left(\delta^{*}+\pi-\tau^{*}\right) \rho^{-2} J_{n}\end{array}$ & \\
\hline $\begin{array}{l}\Psi_{0} \\
0^{-4} \psi_{4}\end{array}$ & -2 & $\begin{array}{l}2\{(\delta-2 \beta \\
2\{(\Delta+2 \gamma \\
+\left(\delta^{*}+3 \pi\right. \\
\text { Notation: }\end{array}$ & $\begin{array}{l}-4 \tau)\left[\left(\delta-\pi^{*}\right) T_{\ell \ell}-\left(D-2 \rho^{*}\right) T_{\ell m}\right]+\left(D-4 \rho-\rho^{*}\right)[(D- \\
+\mu)\left[\left(\Delta-2 \mu-\mu^{*}\right) \rho^{-4} T_{m^{*} m^{*}}-\left(\delta^{*}-2 \pi-2 \beta^{*}-2 \tau^{*}\right) \rho^{-4}\right. \\
\left.-2 \beta^{*}-\tau^{*}\right)\left[\left(\delta^{*}-2 \pi-\tau^{*}\right) \rho^{-4} T_{n n}-\left(\Delta+2 \gamma+2 \mu^{*}-4 \mu\right)\right. \\
D=\ell^{\mu} \partial / \partial x^{\mu}, \\
\rho=-1 /(r-i a \cos \theta), \quad \beta=-\rho^{*} \cot \theta / 2 \sqrt{2}, \\
\tau=-i a \rho \rho^{*} \sin \theta / \sqrt{2}, \quad \mu=\rho^{2} \rho^{*}\left(r^{2}-2 M r+a^{2}\right) / 2, \\
J_{n}=J_{\mu} n^{\mu},\end{array}$ & 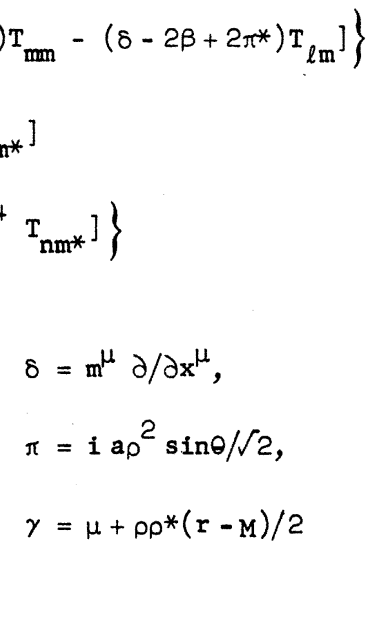 \\
\hline
\end{tabular}

${ }^{a}$ The operator $\Delta$ in this table is not to be confused with $\Delta=r^{2}-2 M r+a^{2}$ used in the body of the paper.

Boyer-Lindquist by $\bar{t}=t+f_{1}(r)+f_{2}(\theta), \bar{\varphi}=\varphi+g_{1}(r)+g_{2}(\theta), \quad r=h(r), \bar{\theta}=j(\theta)$.] In vacuum $(T=0)$ we obtain

$$
\begin{aligned}
& \Delta^{-s} \frac{d}{d r}\left(\Delta^{s+1} \frac{d R}{d r}\right)+\left\{\left[\left(r^{2}+a^{2}\right)^{2} \omega^{2}-4 a M r \omega m+a^{2} m^{2}+2 i a(r-M) m s-2 i M\left(r^{2}-a^{2}\right) \omega s\right] \Delta^{-1}\right. \\
& \left.+2 i r \omega s-A-a^{2} \omega^{2}\right\} R=0 \\
& \frac{1}{\sin \theta} \frac{d}{d \theta}\left(\sin \theta \frac{d S}{d \theta}\right)+\left(a^{2} \omega^{2} \cos ^{2} \theta-\frac{m^{2}}{\sin ^{2} \theta}-2 a \omega s \cos \theta-\frac{2 m s \cos \theta}{\sin ^{2} \theta}-s^{2} \cot ^{2} \theta+s+A\right) S=0 .
\end{aligned}
$$

Equation (8), together with boundary conditions of regularity at $\theta=0$ and $\pi$, constitutes an eigenvalue problem for the separation constant $A$. When $s=0$, the eigenfunctions ${ }_{s} S^{m}{ }_{l}$ are the oblate spheroidal wave functions ${ }^{15} S^{m}{ }_{l}\left(-a^{2} \omega^{2}, \cos \theta\right)$. When $a \omega=0$, the eigenfunctions are the spin-weighted spherical harmonics ${ }^{16}{ }_{s} Y^{m}{ }_{l}(\theta, \varphi)={ }_{s} S^{m}{ }_{l}(\theta) e^{i m \varphi}$, and $A=(l-s)(l+s+1)$. When sources are present $(T \neq 0)$, we can use the eigenfunctions of Eq. (8) to separate Eq. (5) by expanding $T=\sum G(r)_{s} S^{m}{ }_{l}(\theta) e^{i m \varphi} e^{-i \omega t}$.

The asymptotic solutions of Eq. (7) at $r=\infty$ are $e^{i \omega r} / r^{(2 s+1)}$ (outgoing waves) and $e^{-i \omega r} / r$ (ingoing waves). This corresponds to

$$
\begin{aligned}
& \Phi, \varphi_{2}, \psi_{4} \sim e^{i \omega r} / r, \quad \varphi_{0} \sim e^{i \omega r} / r^{3}, \quad \psi_{0} \sim e^{i \omega r} / r^{5} \quad \text { (outgoing); } \\
& \Phi, \varphi_{0}, \psi_{0} \sim e^{-i \omega r} / r, \quad \varphi_{2} \sim e^{-i \omega r} / r^{3}, \quad \psi_{4} \sim e^{-i \omega r} / r^{5} \quad \text { (ingoing). }
\end{aligned}
$$

One identifies $\Phi, \varphi_{2}$, and $\psi_{4}(s \leqslant 0)$ as the outgoing radiative parts of the fields because for outgoing waves they alone die out as $1 / r$; all other Newman-Penrose quantities die out more rapidly. For the 
analogous reason one identifies $\Phi, \varphi_{0}$, and $\psi_{0}(s \geqslant 0)$ as the ingoing radiative parts.

The event horizon is at $r=r_{+}$, the larger root of $\Delta=0$. The solutions at the horizon are $e^{i k r^{*}}$ and $e^{-i k r^{*}} \Delta^{-s}$, where $k=\omega-m a / 2 M r_{+}, d r^{*} / d r=\left(r^{2}+a^{2}\right) / \Delta$, and $r^{*} \rightarrow-\infty$. The factor $\Delta^{-s}$ is not a physical singularity; it arises because the Newman-Penrose field quantities are projections on the tetrad (2), which goes singular at $\Delta=0$. (See Ref. 10 for a discussion of this point in the Schwarzschild case.) The correct boundary condition at the horizon is not that the waves be ingoing in these coordinates ${ }^{17,18}$; rather, they must be ingoing as seen by all physical observers-who are "dragged" with angular velocity $d \varphi / d t=a / 2 M r_{+}$near the horizon. The correct ingoing boundary condition then turns out to be $R \sim e^{-i k r^{*}} \Delta^{-s}$.

Maxwell's equations are sufficiently simple that, when one has solved Eq. (5) for $\varphi_{2}$ say, one can find the complete electromagnetic field, up to an undetermined Coulomb term, by integrating (nonseparable) Pfaffian equations for $\varphi_{1}$ and $\varphi_{0}$ (see Ref. 5). Then the stress-energy tensor is given by

$$
\begin{aligned}
4 \pi T_{\mu \nu}=\left\{\varphi_{0} \varphi_{0}{ }^{*} n_{\mu} n_{\nu}+2 \varphi_{1} \varphi_{1} *\left[l_{(\mu} n_{\nu)}+m_{(\mu} m_{\nu)} *\right]+\varphi_{2} \varphi_{2} * l_{\mu} l_{\nu}-4 \varphi_{0}^{*} \varphi_{1} n_{(\mu} m_{\nu)}\right. & \\
& \left.-4 \varphi_{1} * \varphi_{2} l_{(\mu} m_{\nu)}+2 \varphi_{2} \varphi_{0} m_{\mu} m_{\nu}\right\}+ \text { c.c. }
\end{aligned}
$$

where round brackets on subscripts denote symmetrization. The total energy flux per unit solid angle for outgoing waves at infinity can be found from $\varphi_{2}$ alone:

$$
\frac{d^{2} E}{d t d \Omega}=\lim _{r \rightarrow \infty} \frac{r^{2}}{2 \pi}\left|\varphi_{2}\right|^{2}
$$

The squares of the real and imaginary parts of $\varphi_{2}$ give the amount of energy in the two linear polarization states along the directions $\vec{e}_{\hat{\theta}}$ and $\overrightarrow{\mathrm{e}}_{\hat{\varphi}}$, respectively.

For gravitational waves with $\psi_{4} \propto e^{-i \omega t} / r$,

$$
\frac{d^{2} E}{d t d \Omega}=\lim _{r \rightarrow \infty} \frac{r^{2}}{4 \pi \omega^{2}}\left|\psi_{4}\right|^{2} .
$$

The real and imaginary parts of $\psi_{4}$ describe the linear polarization states along the directions $\overrightarrow{\mathrm{e}}_{\hat{\theta}}$ and $\overrightarrow{\mathrm{e}}_{\hat{\varphi}}$, and $\overrightarrow{\mathrm{e}}_{\hat{\theta}} \pm \overrightarrow{\mathrm{e}}_{\hat{\varphi}}$, respectively. It is likely, but not yet proved, that one can calculate the complete perturbed Riemann tensor $\left(\psi_{0}, \psi_{1}, \psi_{2}, \psi_{3}\right.$, $\psi_{4}$ ) from a knowledge of $\psi_{0}$ or $\psi_{4}$ alone, except for undertermined monopole and dipole terms.

A number of expected results can easily be verified by using Eq. (7). Misner ${ }^{19}$ has proposed that a synchrotronlike mechanism might be responsible for Weber's observations. He has suggested that matter spiraling into a massive rotating black hole at the center of the Galaxy might have its radiation beamed into the galactic plane in high harmonics of the orbital frequency, instead of being generated isotropically. Using the scalar-field analog, it has been found ${ }^{20,18}$ that the beaming is cut down by a barrier integral which is never small for astrophysically reasonable sources. If we take the large- $m$ limit of Eq. (7) (with $\omega=m \times$ angular velocity of source), we obtain exactly the same barrier integral for the scalar, electromagnetic, and gravitational cases at dominant order $\left(m^{2}\right)$. Polarization effects, which differ from one type of field to another, enter only at the next smallest order (order $m$ ). Thus, the barrier-integral conclusions of the scalar analysis are valid for the electromagnetic and gravitational cases.

If $\omega=0, \mathrm{Eq}$. (7) can be solved in terms of hypergeometric functions. There are no solutions which are well-behaved at infinity and at the event horizon. Maxwell's equations then imply (Ref. 5) that $\varphi_{1}=Q \rho^{2}$, which corresponds to adding a constant charge $Q$ to the black hole. This verifies Ipser's argument ${ }^{21}$ that the only stationary electromagnetic perturbation of a rotating black hole corresponds to adding charge. If we had found a solution $\psi_{4}$ with $\omega=0$, this would have implied the existence of a new stationary blackhole solution, which presumably would have violated the combined theorems of Carter ${ }^{22}$ and Hawking. ${ }^{23}$

The "spin down" (loss of angular momentum) of a rotating black hole caused by a stationary, nonaxisymmetric perturbation has been discussed by Press $^{24}$ (scalar perturbation) and Hawking and Hartle $^{25}$ (gravitational perturbation with $a \ll M$ ). It turns out that this problem can be solved from a knowledge of $\varphi_{0}$ or $\psi_{0}$ produced by the perturbation. Details of this calculation will be published elsewhere.

Misner ${ }^{17}$ has recently shown that by "superradiant scattering," waves can extract rotational energy from a black hole. The boundary condition $R \sim e^{-i k r^{*}} \Delta^{-s}$ implies that as waves with $k \omega$ $<0$ go down a black hole, they carry negative "energy at infinity" with themselves. If the radiation to infinity of an orbiting particle were balanced by such negative-energy flow down the 
hole, the particle would be in a "floating orbit." Preliminary calculations ${ }^{26}$ with the scalar equation suggest that the details of super-radiant scattering and floating orbits may depend strongly on the spin of the field. The calculations for electromagnetic and gravitational radiation are now underway.

I thank William H. Press and Kip S. Thorne for helpful discussions.

\footnotetext{
*Work supported in part by the National Science Foundation under Grants No. GP-28027 and No. GP-27304.

${ }^{1} \mathrm{~J}$. Weber, Nuovo Cimento B4, 202 (1971), and references therein.

${ }^{2}$ D. Lynden-Bell and M. J. Rees, Mon. Notic. Roy. Astron. Soc. 152, 461 (1971).

${ }^{3}$ C. Bolton, Bull. Amer. Astron. Soc. 3 , 458 (1971); W. A. Hiltner and J. Werner, Astrophys. J. 175, L19 (1972), and references therein.

${ }^{4}$ R. P. Kerr, Phys. Rev. Lett. 11, 237 (1963).

${ }^{5}$ E. D. Fackerell and J. R. Ipser, Phys. Rev. D 5 , 2455 (1972).

${ }^{6} \mathrm{~S}$. A. Teukolsky, California Institute of Technology Report No. OAP-269, 1971 (to be published).

${ }^{7}$ B. Carter, Commun. Math. Phys. 10, 280 (1968).

${ }^{8}$ D. R. Brill, P. L. Chrzanowski, C. M. Pereira,
}

E. D. Fackerell, and J. R. Ipser, Phys. Rev. D $\underline{5}, 1913$ (1972).

${ }^{9}$ R. H. Boyer and R. W. Lindquist, J. Math. Phys. $\underline{8}$, 265 (1967).

${ }^{10}$ R. H. Price, Phys. Rev. D $\underline{5}, 2419,2439$ (1972).

${ }^{11}$ J. M. Bardeen and W. H. Press, to be published.

${ }^{12}$ E. Newman and R. Penrose, J. Math. Phys. $\underline{3}, 566$ (1962).

${ }^{13}$ W. Kinnersley, J. Math. Phys. 10, 1195 (1969).

${ }^{14}$ C. W. Misner, K. S. Thorne, and J. A. Wheeler, Gravitation (Freeman, San Francisco, 1971).

${ }^{15}$ C. Flammer, Spheroidal Wave Functions (Stanford

Univ. Press, Stanford, 1957).

${ }^{16}$ J. N. Goldberg, A. J. Macfarlane, E. T. Newman,

F. Rohrlich, and E. C. G. Sudarshan, J. Math. Phys. $\underline{8}$, 2155 (1967).

${ }^{17} \mathrm{C}$. W. Misner, to be published.

${ }^{18}$ J. M. Bardeen, W. H. Press, and S. A. Teukolsky, to be published.

${ }^{19}$ C. W. Misner, Phys. Rev. Lett. 28, 994 (1972).

${ }^{20} \mathrm{C}$. W. Misner and P. L. Chzranowski, to be published.

${ }^{21}$ J. R. Ipser, Phys. Rev. Lett. 27, 529 (1971).

${ }^{22}$ B. Carter, Phys. Rev. Lett. 26, 331 (1971).

${ }^{23}$ S. W. Hawking, Commun. Math. Phys. 25, 152 (1972).

${ }^{24}$ W. H. Press, Astrophys. J. 175, $243(1972)$.

${ }^{25} \mathrm{~S}$. W. Hawking and J. B. Hartle, to be published.

${ }^{26}$ W. H. Press and S. A. Teukolsky, Nature (London) $\underline{238}, 211$ (1972).

\title{
Evidence for Direct Emission in the Decay $K^{ \pm} \rightarrow \pi^{ \pm} \pi^{0} \gamma$
}

\author{
R. J. Abrams,* A. S. Carroll, T. F. Kycia, K. K. Li, J. Menes, 市 \\ D. N. Michael, P. M. Mockett, and R. Rubinstein \\ Brookhaven National Laboratory, Upton, New York 11973
}

(Received 3 August 1972)

\begin{abstract}
We have obtained about 4000 events above background which satisfy the two-constraint fit with the hypothesis $K^{ \pm} \rightarrow \pi^{ \pm} \pi^{0} \gamma$. Their Dalitz-plot population shows the existence of direct radiation in addition to that of the expected inner bremsstrahlung. The best fit to the sum of $\mathrm{K}^{+}$and $\mathrm{K}^{-}$decays in the charged-pion kinetic energy interval of 55 to $90 \mathrm{MeV}$ gives a branching ratio for direct emission of $(1.56 \pm 0.35) \times 10^{-5}$, with a systematic uncertainty of $\pm 0.5 \times 10^{-5}$. No interference is required for a good fit.
\end{abstract}

It has been apparent for some time that the $\pi^{ \pm} \pi^{0} \gamma$ decay of the $K^{ \pm}$meson could have a direct emission (DE) contribution of the same order as the inner bremsstrahlung part (IB). ${ }^{1,2}$ Such DE would be a new type of decay of the $K$ meson since the IB part necessarily follows from the $K_{\pi 2}$ decay. Several authors ${ }^{3}$ have attempted to calculate this direct amplitude, and others ${ }^{4}$ have looked for such a contribution to the $\pi^{ \pm} \pi^{0} \gamma$ decay. A direct decay into $\pi^{ \pm} \pi^{0} \gamma$ could proceed without violation of the $\Delta I=\frac{1}{2}$ rule, which suppresses by a factor of $\sim 500$ the $K_{\pi 2}$ rate and hence the IB rate. For this reason the $K^{ \pm} \rightarrow \pi^{ \pm} \pi^{0} \gamma$ decay mode has been considered to have excellent prospects of exhibiting direct emission. This decay rate has also been suggested as one in which $C P$ nonconservation could possibly be observed. ${ }^{5}$

We have studied the $\pi^{ \pm} \pi^{0} \gamma$ decay mode from kaon decays in flight in an experiment carried out in a $1.8-\mathrm{GeV} / c$ partially separated kaon beam at the Brookhaven National Laboratory (BNL) alternating gradient synchrotron. A schematic of the setup is shown in Fig. 1. The incident kaon and its charged decay pion were recorded with a 